\title{
Lymphadenitis associated with cat-scratch disease simulating a neoplasm: Imaging findings with histopathological associations
}

\author{
YING CHEN $^{1 *}$, YAN-BIAO FU ${ }^{2 *}$, XIU-FANG XU ${ }^{3}$, YAO PAN ${ }^{1}$, CHEN-YING LU $^{1}$, \\ XIU-LIANG ZHU ${ }^{1}$, QING-HAI LI ${ }^{1}$ and RI-SHENG YU ${ }^{1}$
}

Departments of ${ }^{1}$ Radiology and ${ }^{2}$ Pathology, Second Affiliated Hospital, Zhejiang University School of Medicine, Hangzhou, Zhejiang 310009; ${ }^{3}$ Department of Radiology, Hangzhou Medical College, Hangzhou, Zhejiang 310053, P.R. China

Received August 31, 2016; Accepted September 28, 2017

DOI: $10.3892 / \mathrm{ol} .2017 .7311$

\begin{abstract}
The lymphadenitis associated with cat-scratch disease (CSD) is often confused with neoplasms by a number of radiologists and clinicians, and consequently, unnecessary invasive procedures or surgeries are performed. In the present study, the contrast-enhanced computed tomography (CT) and magnetic resonance imaging (MRI) findings of 10 patients (6 men and 4 women) with clinically and pathologically confirmed lymphadenitis associated with CSD were retrospectively analyzed (CT in 3 patients, MRI in 6 patients, and CT and MRI in 1 patient) at The Second Affiliated Hospital of Zhejiang University School of Medicine (Hangzhou, China) between January 2007 and November 2014. As a result, 17 enlarged lymph nodes were identified in 10 cases. The 5 nodes identified by CT scan exhibited relatively inhomogeneous isodensity to muscle, with patchy low density in the center. All 14 nodes identified by MRI scan exhibited homogeneous or heterogeneous isointensity to muscle or slightly increased intensity compared with that of muscle on T1-weighted images (T1WI), and homogeneous or heterogeneous hyperintensity on fat-suppressed T2WI. Following enhancement, all 17 enlarged lymph nodes associated with CSD demonstrated the following 3 different enhancement patterns: Moderate homogeneous enhancement $(n=8)$, which was associated with histologically identified early disease stage; marked heterogeneous enhancement with no enhancement of the necrotic areas $(n=4)$, and heterogeneous enhancement with progressively 'spoke-wheel-like' (defined as radiating enhancement from the center) enhancement of the patchy low-density area $(n=1)$, which was associated with histologically identified intermediate
\end{abstract}

Correspondence to: Dr Ri-Sheng Yu, Department of Radiology, Second Affiliated Hospital, Zhejiang University School of Medicine, 88 Jiefang Road, Hangzhou, Zhejiang 310009, P.R. China

E-mail: cjr.yurisheng@vip.163.com

*Contributed equally

Key words: lymphadenitis, cat-scratch disease, computed tomography, magnetic resonance imaging, pathology disease stage; and astral low-density/hypointensity with marked enhancement $(n=2)$ or a 'rose flower' $\operatorname{sign}(n=2)$, which was associated with histologically identified late disease stage. We hypothesized that the CT and MRI results of lymphadenitis in CSD may be associated with the pathological features. It may be suggested that the diagnosis of CSD may be formed when considering the characteristic CT and MRI features of astral low-density/hypointensity with marked enhancement or a 'rose flower' sign (defined as marginal petaloid enhancement) in the late disease stage, or the MRI results of homogeneous, moderate enhancement in the early disease stage, or the CT/MRI data of heterogeneous enhancement with non-enhancing area in the center in the intermediate disease stage, in solitary or multiple enlarged lymph nodes associated with general subcutaneous edema in the vicinity of the nodes on CT/MRI and with a history of cat exposure.

\section{Introduction}

Cat-scratch disease (CSD) is an infrequent infection caused by Bartonella henselae, a Gram-negative, facultative intracellular bacillus acquired from exposure to cats or cat fleas (1-3). Bartonella henselae are able to contaminate feline saliva and infect humans through scratching or biting (4), with the lowest incidence occurring in mountainous, arid regions and the highest incidence occurring in the humid, warm climate of the South of the United States (5). As Bartonella species are difficult to culture, the serological study of specific immunoglobulin ( $\mathrm{Ig}$ ) $\mathrm{G}$ is generally recommended in order to obtain a CSD diagnosis. A positive $\operatorname{IgM}$ test indicates acute infection; however, the production of $\operatorname{IgM}$ antibodies only occurs over a very short period of time is rarely detected in CSD (6).

The typical manifestation of CSD is a regional lymphadenitis, which presents in $\sim 90 \%$ of cases as a subacute regional lymphadenopathy (7), and it occurs predominantly in children and young adults (8). Although the lymphadenitis usually spontaneously resolves, or may be treated with a short course of antibiotics (9-11), it occasionally progresses to severe life-threatening complications. Atypical CSD may present with a wide range of clinical manifestations, including neuroretinitis, periadenitis, hepatosplenic lesions, osteomyelitis, endocarditis, encephalitis and fevers of unknown origins (12-14), and occurs in 5\% of immunocompetent 
patients. Additionally, the lymphadenitis associated with CSD is often confused with neoplasms by a number of radiologists and clinicians, as they are not familiar with this pseudo-tumor lesion (15). Therefore, the early consideration of this disorder will avoid unnecessary invasive procedures or surgery.

The estimated annual incidence of CSD in the United States is 4.5-9.3 outpatient diagnoses per 100,000 individuals and 0.19-0.86 hospital admissions per 100,000 individuals $(16,17)$. However, a review of the English literature revealed that $<20$ cases of lymphadenitis associated with CSD on contrast-enhanced computed tomography (CT) and magnetic resonance imaging (MRI) data have been well addressed worldwide $(15,18-20)$ since its first description in 1889 by Parinaud (21), and the majority of these studies were of sporadic cases and small case series. Additionally, no distinguishable early, intermediate or late stage imaging manifestations or pathological features of CSD have been identified.

The present study highlights the predominant radiological and pathological features in 10 cases of regional lymphadenitis associated with CSD, focusing on the association between the early, intermediate and late stages of the imaging results, and the pathological features of each stage, which, to the best of our knowledge, have not been described in-depth previously in the English literature.

\section{Patients and methods}

Patients. The present study was approved by the Institutional Research and Ethics Board of The Second Affiliated Hospital of Zhejiang University School of Medicine (Hangzhou, China) and the requirement for patient informed consent was waived. Enhanced CT $(n=3)$, enhanced MRI $(n=6)$, combined enhanced CT and MRI $(n=1)$, clinical $(n=10)$ and pathological $(n=10)$ features of 10 cases of CSD were retrospectively reviewed at The Second Affiliated Hospital of Zhejiang University School of Medicine between January 2007 and November 2014. The differences in the diagnostic techniques used are due to the retrospective nature of the study. Among the 10 patients, there were 6 men and 4 women, with ages ranging from 18 to 57 years (mean, 40.9 years). The duration of symptoms ranged from 7 days to 8 years.

The clinical signs and symptoms included painless nodes $(n=5)$, painful nodes $(n=3)$, painful swelling $(n=3)$ and mild fever $(n=2)$. A total of 2 patients without any complaints were incidentally identified by a routine physical examination. A total of 7 patients had pet cats and 3 patients had pet dogs, but they all initially denied a history of cat or dog scratches. Physical examinations were unremarkable, with the exception of tenderly mobile nodular lesions with a smooth surface and slight tenderness at the elbow $(n=6)$, neck $(n=2)$, groin $(n=1)$ and axilla $(n=1)$. Laboratory tests indicated leukocytosis $\left(11.4 \times 10^{9} / 1\right.$ and $12.8 \times 10^{9} / 1$; normal range, $\left.4.0-10.0 \times 10^{9} / 1\right)$ and slightly elevated C-reactive protein $(20.5$ and $31.8 \mathrm{mg} / \mathrm{l}$; normal range, $<10.0 \mathrm{mg} / \mathrm{l}$ ) in 2 cases. No evidence of peripheral eosinophilia was identified. Blood cultures and viral serological tests were all negative. Tuberculin tests (purified protein derivative test) were all negative. The expression levels of various tumor markers (carcinoembryonic antigen, $\alpha$-fetoprotein, carbohydrate antigen (CA)19-9, CA125, CA242, neuron-specific enolase, squamous cell carcinoma antigen, prostate-specific antigen and $\beta$-human chorionic gonadotrophin) were all negative and no primary malignancy was identified in all 10 patients. No serological or skin tests for CSD were performed.

Radiological examination. CT examinations were performed in 4 patients using 16-row CT scanners (Siemens Somatom Sensation 16; Siemens AG, Munich, Germany) with the following parameters: $6-\mathrm{mm}$ axial sections ranging from the plane of the ilium to the last plane of the inferior pubic ramus for the pelvic cavity in 1 patient; 4-mm axial sections ranging from the plane of the mandible to the last plane of the thyroid cartilage for the neck in 1 patient; 6 -mm axial sections ranging from the plane of the clavicle to the last plane of the shoulder blade for the axilla in 1 patient; and 5-mm axial sections ranging from the plane of the middle humerus to the last plane of the middle ulna for the elbow in 1 patient. All patients were examined in a fasting state with plain scanning at first, and then an $80 \mathrm{ml} /$ bolus injection of non-ionic contrast medium (OMNIPAQUE 300; GE Healthcare, Chicago, IL, USA) was administered via the antecubital vein for multiple-phase enhanced scanning.

MRI examinations were performed using a $1.5 \mathrm{~T}$ or $3.0 \mathrm{~T}$ magnet (SIGNA ${ }^{\mathrm{TM}}$; GE Healthcare). The elbows in 6 patients and the neck in 1 patient all underwent spin-echo (SE) T1-weighted imaging (T1WI), turbo SE (TSE) fat-suppression T2WI, and axial, coronal and sagittal contrast-enhanced T1WI (Omniscan $0.1 \mathrm{mmol} / \mathrm{kg}$ body weight; GE Healthcare, Chicago, IL, USA) with fat suppression. The imaging parameters of the elbow scans were as follows: Repetition time/echo time (TR/TE) of T1WI, 240-700/3.6-13 msec; and TR/TE of T2WI, 2,840-2,900/72-85 msec. The matrix was 256x256, the number of excitations (NEX) was 1.0-2.0, the field-of-view (FOV) was 140x140-180x200 mm, and slice thickness was $4.0 \mathrm{~mm}$, with a $0.4-0.5 \mathrm{~mm}$ interslice gap. The imaging parameters of the neck scans were as follows: T1WI TR/TE, 400-600/8-9.9 msec; T2WI TR/TE, 4,020-4,820/84.8 msec; NEX, 2.0-3.0; 256x256 matrix; FOV, 140x140-180x200 mm; and slice thickness, $4.0 \mathrm{~mm}$, with no interslice gap.

Image analysis. All CT and MRI images were reviewed separately by 2 radiologists who had no knowledge of the clinical and pathological outcomes. Discordance between opinions was resolved by consensus. The CT and MRI evaluation included the site, number, size, density or signal intensity and the enhancement patterns of the loco-regional lesions, and the existence of strands in the surrounding soft tissue.

Pathological analysis. The pathological specimens of lymph node tissues were obtained by resection in 6 patients and by percutaneous needle biopsy in 4 patients. The lymph node tissue samples were fixed with $10 \%$ neutral formaldehyde solution for $24 \mathrm{~h}$ at $18^{\circ} \mathrm{C}$, embedded in paraffin and cut into $3-\mu$ m-thick sections. The sections were subsequently stained with routine hematoxylin and eosin for $2 \mathrm{~h}$ at $18^{\circ} \mathrm{C}$ prior to being dehydrated with $95 \%$ alcohol 5 min (4 times) and xylene 5 min (twice), and sealed with neutral balsam. Subsequently, the structure of the tissue cells was observed at $\mathrm{x} 200$ magnification using a BX50 Olympus microscope (Olympus Corporation, Tokyo, Japan). 


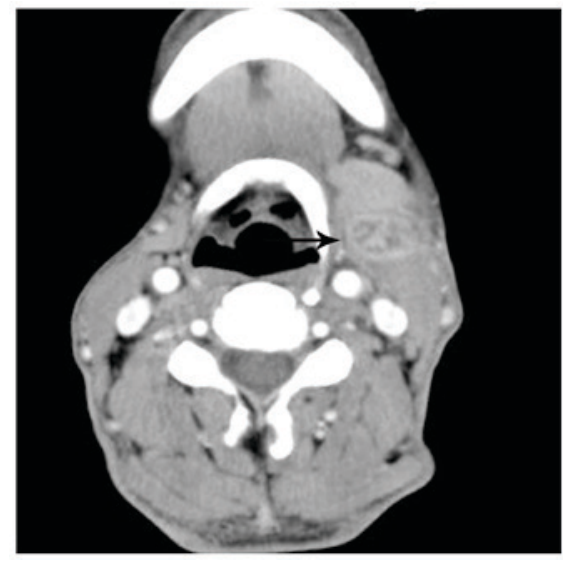

Figure 1. Lymphadenopathy in a 57-year-old man with cat-scratch disease at the intermediate stage in the left neck. The node (arrow) displays heterogeneous enhancement with 'spoke-wheel-like' enhancement of the patchy low-density area on an enhanced computed tomography scan following intravenous contrast administration.

All specimen analyses were confirmed by an experienced pathologist for diagnostic accuracy. The histological staging of the lesions and the association between the enhanced imaging data and the pathological features were evaluated in each case, with a particular focus on the coagulation or liquid necrosis, astral abscess and the presence of fibrosis.

\section{Results}

Imaging data. In total, there were 17 enlarged lymph nodes identified in 10 cases, of which 12 nodes were scanned on enhanced MRI, 3 on enhanced CT, and 2 on enhanced CT and MRI. Among them, 13 nodes were located at the elbow, 2 in the neck, 1 in the groin and 1 in the axilla. Multiple lesions were identified in 4 cases, and singular lesions in 6 patients. All the lesions were round, well-delineated nodes or nodules. The size of the enlarged lymph nodes ranged from $0.8-3.0 \mathrm{~cm}$. On the CT scans, all the 5 nodes identified exhibited relatively inhomogeneous isodensity to muscle, with patchy or astral low density in the center. Following enhancement, 2 of the 5 nodes demonstrated marked heterogeneous enhancement, with no enhancement of the patchy low-density central areas, 1 of the 5 nodes displayed heterogeneous enhancement with progressive 'spoke-wheel-like' (defined as radiating enhancement from the center) enhancement of the patchy low-density area (Fig. 1), and the other 2 nodes demonstrated heterogeneous enhancement with peripheral flower ring reinforcement of the astral low-density area, also known as the 'rose flower' sign.

On the MRI scans, all the 14 nodes identified were homogeneously or heterogeneously isointense to muscle or with slightly increased intensity compared with that of muscle on T1WI, and homogeneously or heterogeneously hyperintense on fat-suppressed T2WI. Subsequent to enhancement, 8 of the 14 nodes displayed moderately homogeneous enhancement with no necrotic areas (Fig. 2) and 2 of the 14 nodes demonstrated marked heterogeneous enhancement with no enhancement of the necrotic central areas, which demonstrated patchy hypointense on T1WI and patchy hyperintense on T2WI in the center (Fig. 3A-C). Another 2 of the 14 nodes exhibited

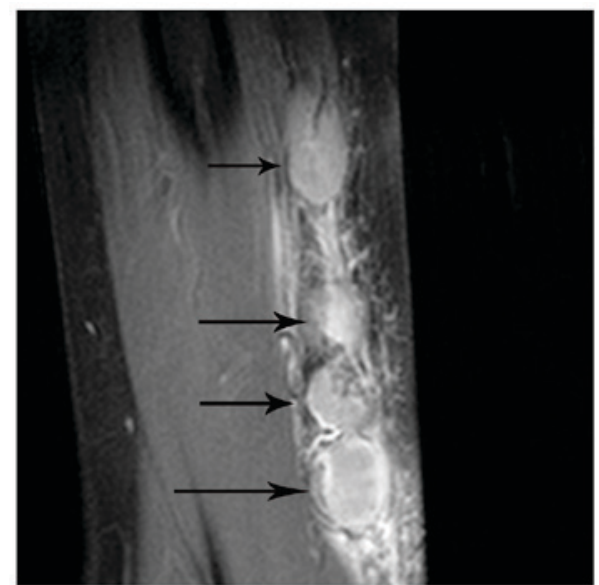

Figure 2. Multiple lymph node involvement in a 50-year-old man with cat-scratch disease at the early stage in the left elbow. The nodes (arrows) exhibit moderately homogeneous enhancement with no necrotic areas on an enhanced fat-suppressed sagittal T1-weighted imaging scan.
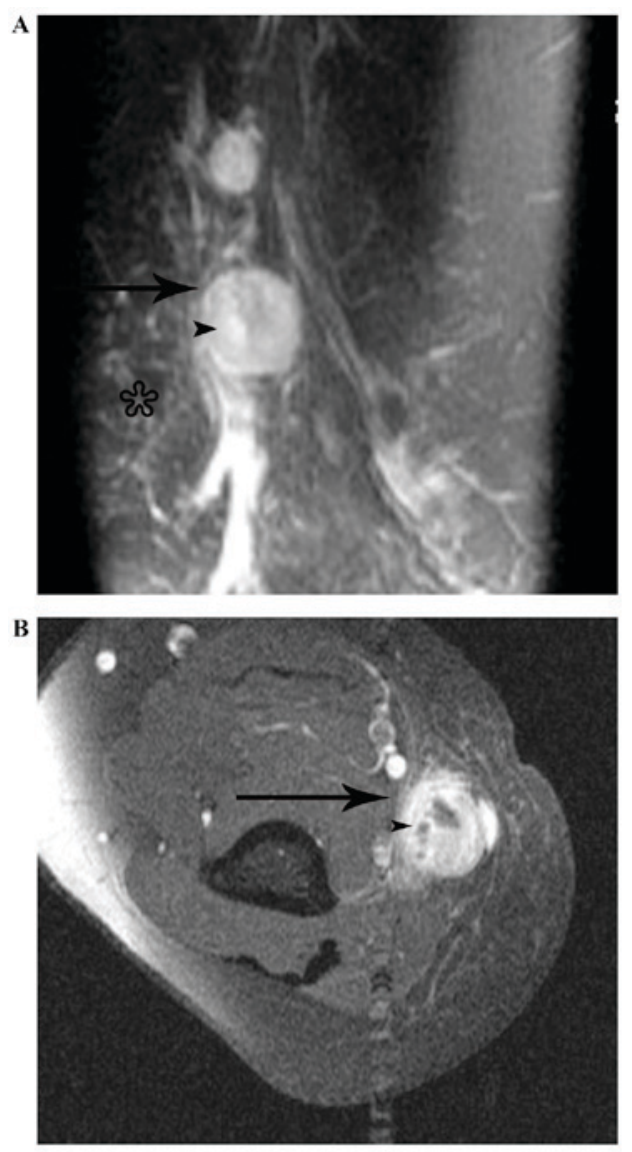

Figure 3. Lymphadenopathy in a 51-year-old woman with cat-scratch disease at the intermediate stage in the left elbow. (A) The node (arrow) indicates heterogeneous hyperintensity with patchy hyperintensity (arrow head) on a fat-suppressed coronal T2-weighted imaging scan, with general subcutaneous edema (star) in the vicinity of the node. (B) Following enhancement, the node demonstrates marked heterogeneous enhancement with no enhancement of the necrotic central areas (arrowhead) on an enhanced fat-suppressed axial T1-weighted imaging scan.

heterogeneous enhancement with marked enhancement of the astral hypointense area on T1WI and T2WI (Fig. 4A-C), and the other 2 exhibited heterogeneous enhancement with 

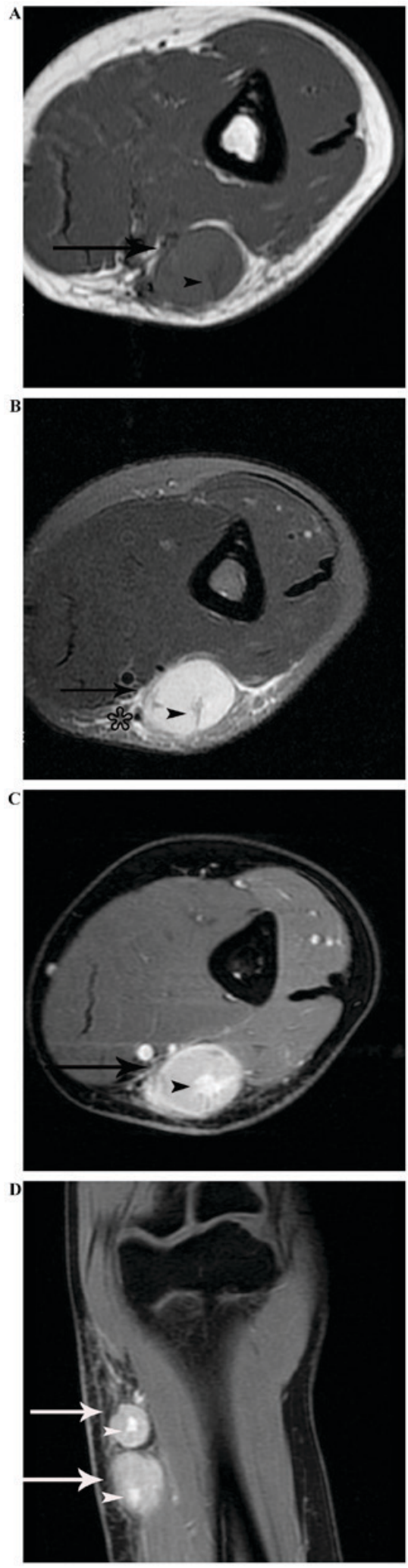

Figure 4. Lymphadenopathy in an 18-year-old man with cat-scratch disease at the late stage in the left elbow. (A) The node (arrow) exhibits heterogeneous isointensity to muscle with astral hypointensity (arrowhead) on T1WI and (B) heterogeneous hyperintensity with astral hypointensity (arrowhead) on fat-suppressed T2WI, with general subcutaneous edema (star) in the vicinity of the node. (C) Following enhancement, the node (black arrow) exhibits heterogeneous enhancement with marked enhancement of the astral hypointense area (black arrowhead) on an enhanced fat-suppressed axial T1-weighted imaging scan. (D) The two nodes (white arrow) demonstrate heterogeneous enhancement with marked enhancement of the astral hypointense area (white arrowhead) on an enhanced fat-suppressed coronal T1-weighted imaging scan. marginal petaloid enhancement of the astral hypointense area, namely the 'rose flower' sign (Fig. 5A-C). In addition, all 10 cases demonstrated general subcutaneous edema in the vicinity of the nodes.

Pathological features. There were 3 stages of CSD histologically, including early stage $(n=3)$, intermediate stage $(n=4)$ and late stage $(n=3)$. In the early stage, there were numerous granulomas with proliferation of histiocytes, lymphoid follicles and neutrophilic aggregation (Fig. 6). In the intermediate stage, there were irregular granulomas with stellate abscesses composed of marked central necrosis surrounded by an inner layer of palisading histiocytes, an intermediate lymphocytic rim, and an outermost zone of fibrosis (Fig. 7). In the late stage, the inflammatory and necrotic regions were replaced or surrounded by the spindle collagen-producing cells, which were composed of variable amounts of fibroblasts or fibrocytes (Fig. 8).

Enhanced imaging data associated with the pathological features. There were 3 types of CT and MRI enhancement patterns in the 17 enlarged lymph nodes associated with CSD. The different enhancement patterns were associated with different pathological features (Tables I-III). According to Tables I-III, the 8 (47.1\%) nodes with moderately homogeneous enhancement (all on MRI) belonged to the histologically determined early stage (stage total, $47.1 \%$ ); the 4 (23.5\%) nodes with marked heterogeneous enhancement with no enhancement of the necrotic central areas ( 2 on CT and 2 on MRI) and heterogeneous enhancement with progressively spoke-wheel-like enhancement of the patchy low-density area in 1 (5.9\%) node (on CT) belonged to the histologically determined intermediate stage (stage total, 29.4\%); and the 2 (11.8\%) nodes with astral low-density/hypointensity with marked enhancement (both on MRI) or the 'rose flower' sign in 2 (11.8\%) nodes (both on $\mathrm{CT} / \mathrm{MRI}$ ) belonged to the histologically determined late stage (stage total, 23.6\%).

\section{Discussion}

CSD is an infrequent zoonotic infectious illness characterized by regional lymphadenopathy and usually caused by the Rickettsia-like microorganism termed Bartonella (formerly Rochalimaa) henselae, a gram-negative coccobacillus $(18,22)$. The condition is most commonly diagnosed in individuals between the ages of 5 and 21 years $(18,23)$. In the present study, there was only 1 patient belonging to this age group, which was not compatible with other studies in the literature. The majority of patients with the disease confirmed recent contact with a cat, usually a kitten, however, occasionally it was not identified by the patient initially. In the present study, although 7 patients had pet cats and 3 patients had pet dogs, none of them recalled this characteristic history prior to surgery or biopsy. Therefore, it is extremely important to obtain a detailed disease history prior to diagnoses.

The clinical manifestation of CSD is variable, with the majority of cases exhibiting non-specific symptoms, such as papules or pustules appearing at the site of the scratch between 7 and 12 days after exposure, which usually heal spontaneously in days to weeks. During the next 1-3 weeks, 

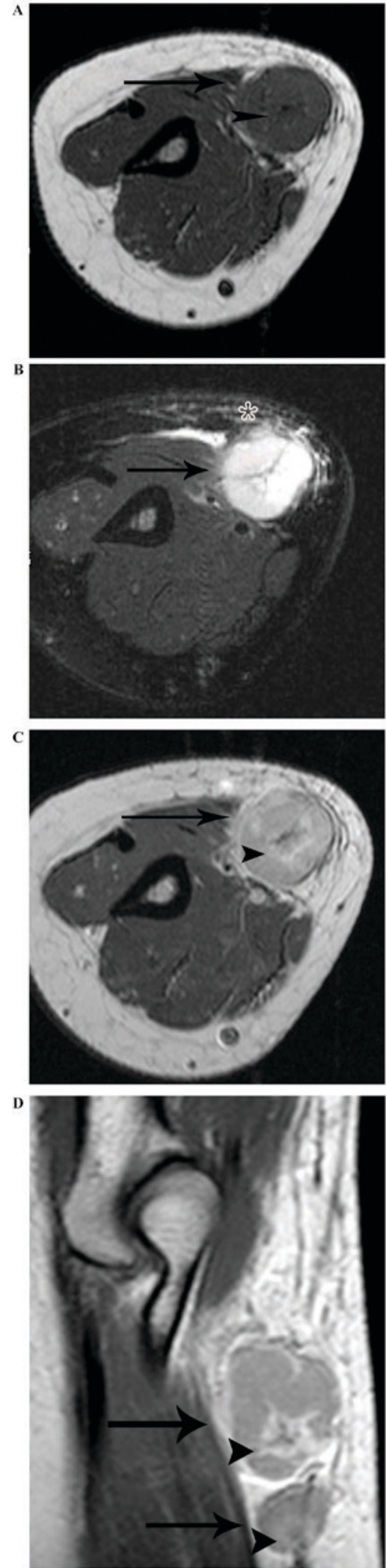

Figure 5. Lymphadenopathy in a 37-year-old woman with cat-scratch disease at the late stage in the right elbow. (A) The node (arrow) demonstrates heterogeneous isointensity to muscle with astral hypointensity (arrowhead) on T1WI, and (B) heterogeneous hyperintensity with astral hypointensity (arrowhead) on fat-suppressed T2WI, with general subcutaneous edema (star) in the vicinity of the node. (C) Following enhancement, the node (arrow) demonstrates heterogeneous enhancement with peripheral flower ring reinforcement of the astral hypointense area (arrowhead) on an enhanced axia T1-weighted imaging scan. (D) The two nodes (arrow) exhibit heterogeneous enhancement with peripheral flower ring reinforcement of the astral hypointense area (arrowhead), on an enhanced sagittal T1-weighted imaging scan.

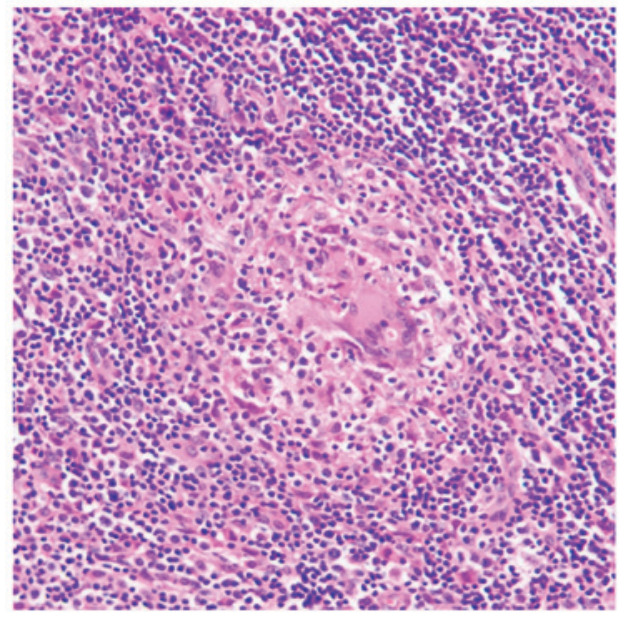

Figure 6. Histological microscopy indicates that there are numerous granulomas with proliferation of histiocytes, lymphoid follicles and neutrophilic aggregation in the early stage of cat-scratch disease (hematoxylin and eosin staining; magnification, x200).

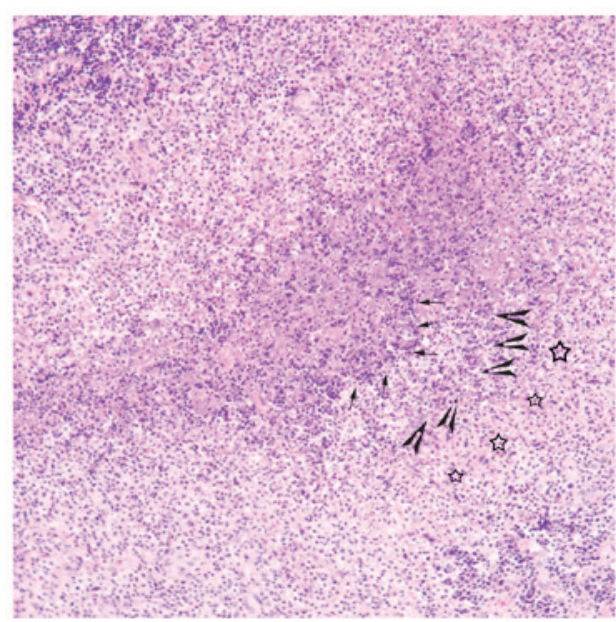

Figure 7. Histological microscopy indicates that there are irregular granulomas with stellate abscesses composed of marked central necrosis surrounded by an inner layer of palisading histiocytes (arrow), an intermediate lymphocytic rim (arrowhead) and an outermost zone of fibrosis (star) in the intermediate stage of cat-scratch disease (hematoxylin and eosin staining; magnification, x100).

the enlargement of regional lymph nodes occurs. On the basis of the frequency of scratches, the involved lymph nodes are situated at upper extremities (46\%), neck (26\%), groin (17\%) or other locations $(24,25)$. The medial epitrochlear region is the most prevalent location $(18,20)$. In the patients of the present study, 6/10 cases of CSD were located at the elbow, probably due to a high frequency of scratches on the hands. A total of 2 patients exhibited lymphadenopathy in the neck, 1 in the inguinal region and the other in the axilla, results which were in accordance with those of previous studies $(24,25)$. The majority of enlarged lymph nodes often regress over weeks to months, and rarely may persist for up to 2 years $(25,26)$. In the present study, the duration of enlarged lymph nodes ranged from 7 days to 8 years, which was the longest recorded time to persist. Regional lymphadenitis may be accompanied by systemic symptoms, including malaise and mild fever (20). 

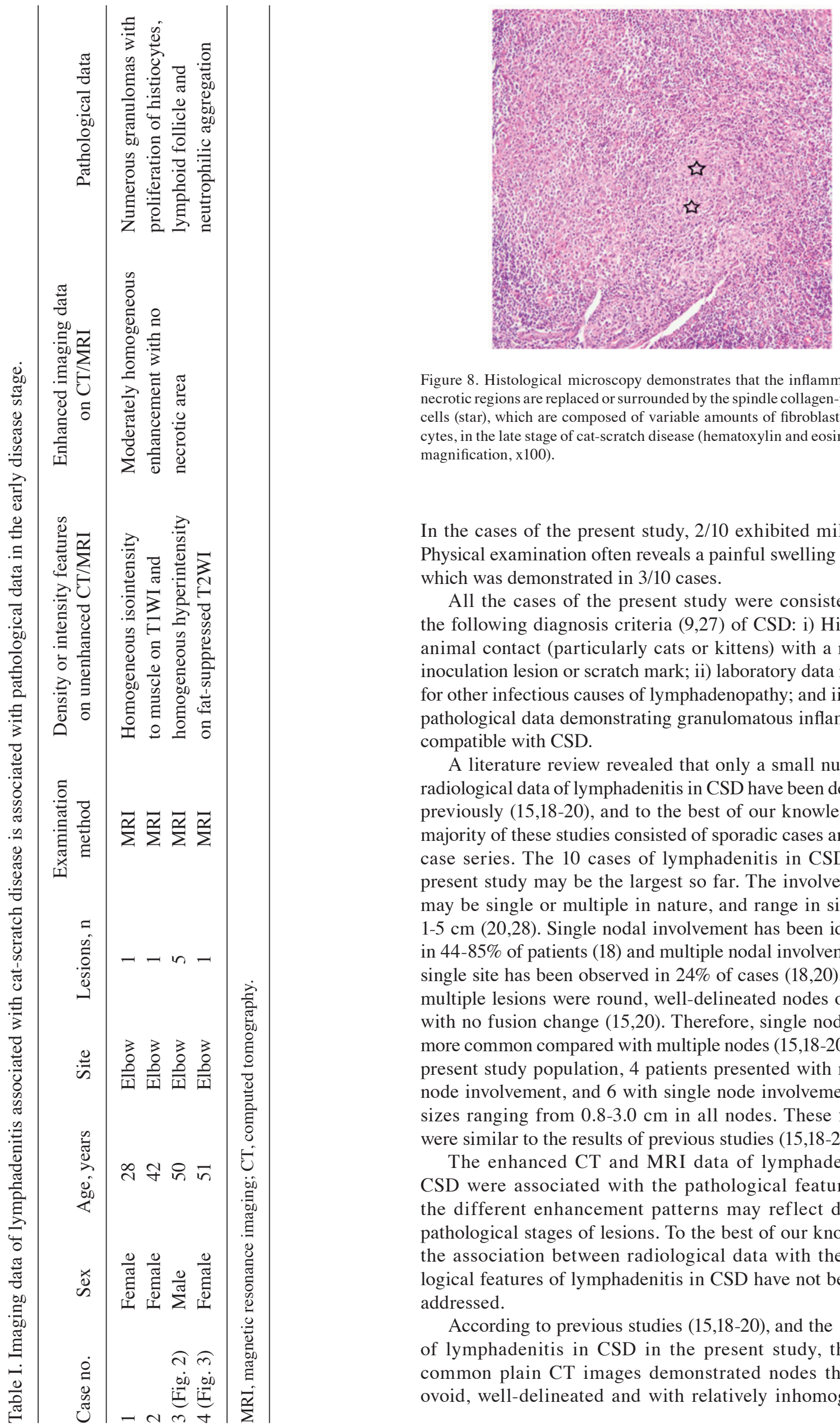

Figure 8 . Histological microscopy demonstrates that the inflammatory and necrotic regions are replaced or surrounded by the spindle collagen-producing cells (star), which are composed of variable amounts of fibroblasts or fibrocytes, in the late stage of cat-scratch disease (hematoxylin and eosin staining; magnification, $\mathrm{x} 100$ ).

In the cases of the present study, 2/10 exhibited mild fever. Physical examination often reveals a painful swelling or node, which was demonstrated in 3/10 cases.

All the cases of the present study were consistent with the following diagnosis criteria $(9,27)$ of CSD: i) History of animal contact (particularly cats or kittens) with a regional inoculation lesion or scratch mark; ii) laboratory data negative for other infectious causes of lymphadenopathy; and iii) histopathological data demonstrating granulomatous inflammation compatible with CSD.

A literature review revealed that only a small number of radiological data of lymphadenitis in CSD have been described previously $(15,18-20)$, and to the best of our knowledge, the majority of these studies consisted of sporadic cases and small case series. The 10 cases of lymphadenitis in CSD of the present study may be the largest so far. The involved nodes may be single or multiple in nature, and range in size from $1-5 \mathrm{~cm}(20,28)$. Single nodal involvement has been identified in $44-85 \%$ of patients (18) and multiple nodal involvement at a single site has been observed in $24 \%$ of cases $(18,20)$. All the multiple lesions were round, well-delineated nodes or nodes with no fusion change $(15,20)$. Therefore, single nodes were more common compared with multiple nodes $(15,18-20)$. In the present study population, 4 patients presented with multiple node involvement, and 6 with single node involvement, with sizes ranging from $0.8-3.0 \mathrm{~cm}$ in all nodes. These findings were similar to the results of previous studies $(15,18-20)$.

The enhanced CT and MRI data of lymphadenitis in CSD were associated with the pathological features, and the different enhancement patterns may reflect different pathological stages of lesions. To the best of our knowledge, the association between radiological data with the pathological features of lymphadenitis in CSD have not been well addressed.

According to previous studies $(15,18-20)$, and the 10 cases of lymphadenitis in CSD in the present study, the most common plain CT images demonstrated nodes that were ovoid, well-delineated and with relatively inhomogeneous 


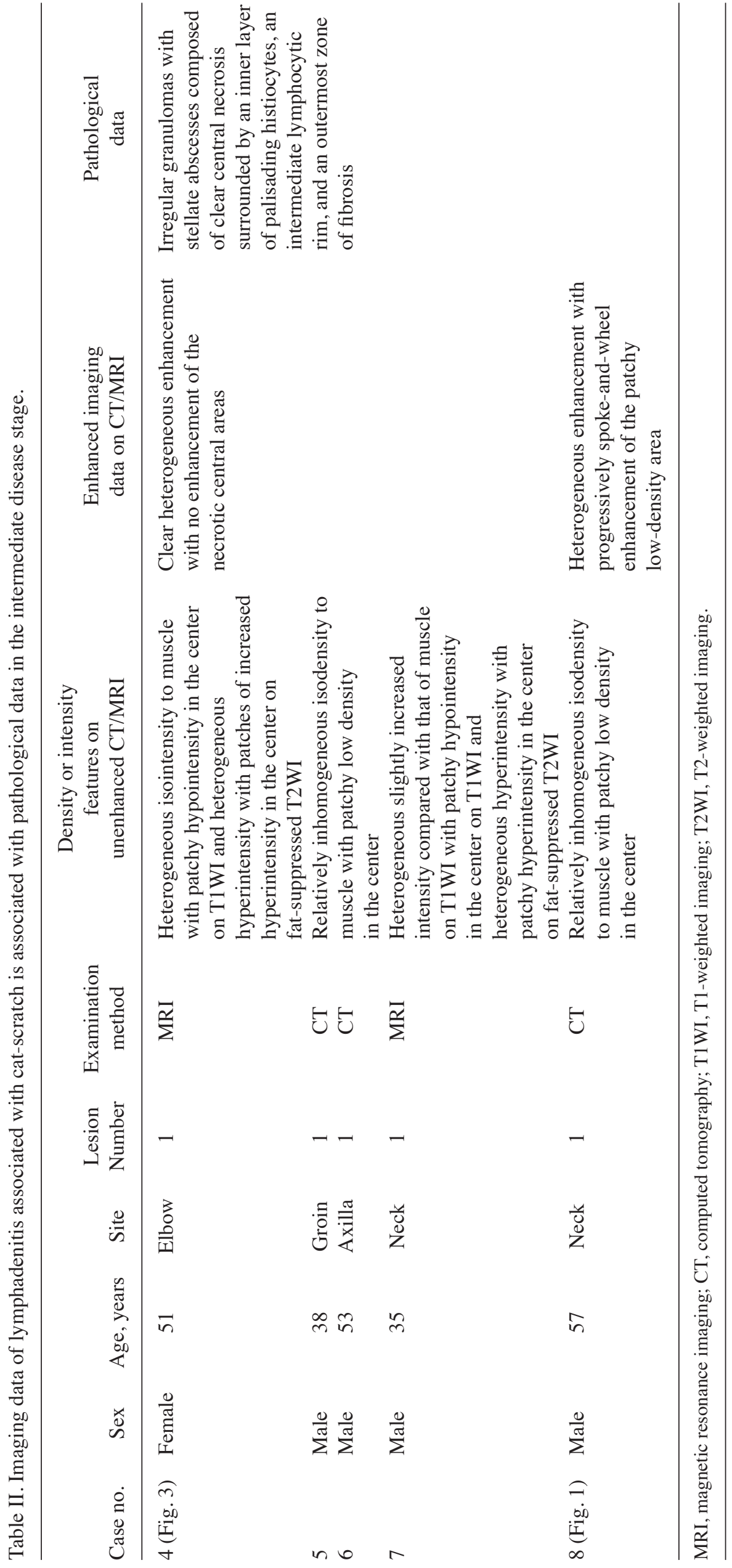




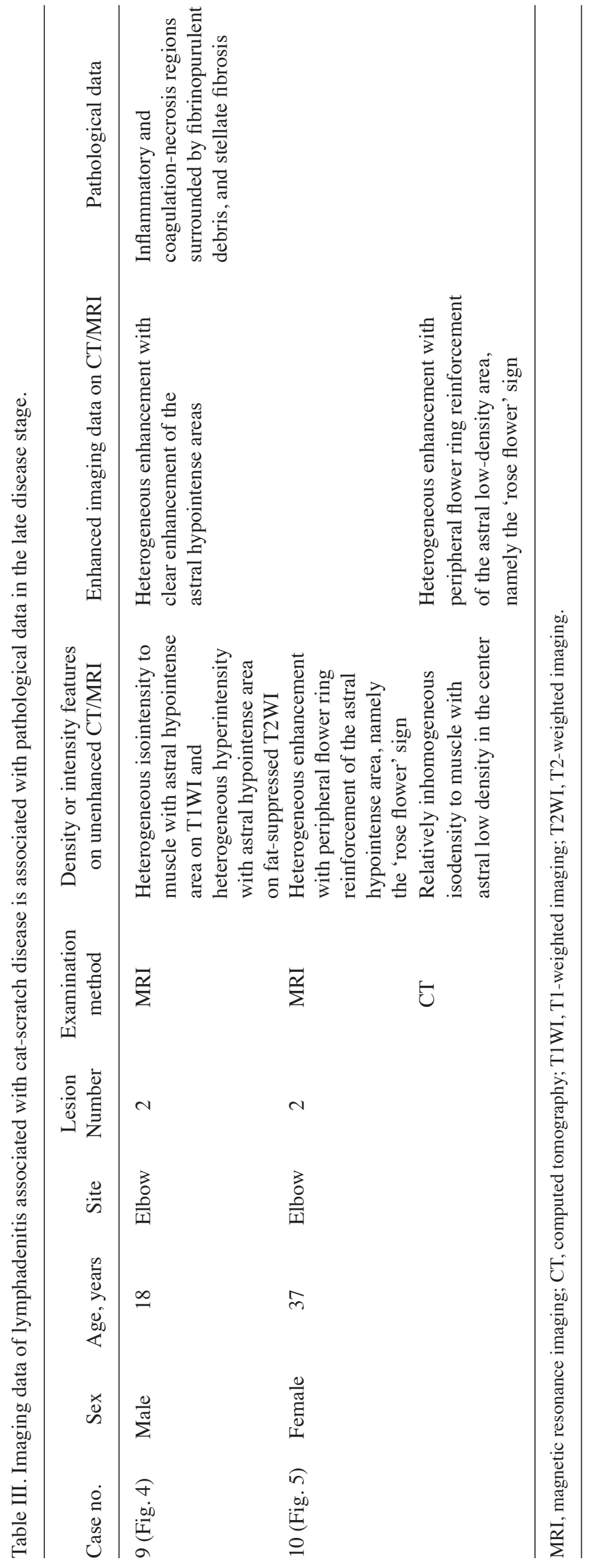


isodensity to muscle, with patchy low density in the center The corresponding unenhanced MRI revealed nodes that were heterogeneously isointense to muscle or with slightly increased intensity compared with that of muscle on T1WI scans, with patchy hypointensity in the center or periphery, and heterogeneous hyperintensity with patches of increased hyperintensity in the center or intermediate signal intensity with patchy hyperintensity in the periphery on fat-suppressed T2WI. Following intravenous contrast administration, the peripheral or central areas demonstrated marked enhancement on the plain $\mathrm{CT}$, whereas on the enhanced CT image, the central or peripheral areas indicated no enhancement at all, or heterogeneous enhancement with progressive 'spoke-wheel-like' enhancement of the patchy low-density central area. The corresponding histological analysis demonstrated irregular granulomas with stellate abscesses composed of marked central necrosis, surrounded by an inner layer of palisading histiocytes, an intermediate lymphocytic rim and an outermost zone of fibrosis. This pattern was observed in $5 / 17(29.4 \%)$ nodes in the present study, and we hypothesized that the non-enhancing areas of the scanning images corresponded to areas of liquid necrosis, and that they belonged to the intermediate disease stage, as identified histologically. Although it was difficult to differentiate CSD at this point from tuberculous granuloma with liquid necrosis by radiological data (29), if associated with the special disease history of cat exposure, it may be diagnosed correctly at a relatively early time-point. It may be that heterogeneous enhancement with progressive 'spoke-wheel-like' enhancement of the central patchy low-density area on CT images is the characteristic manifestation of lymphadenitis in CSD, which has not been well addressed previously to the best of our knowledge, and requires additional cases for verification.

The second most common radiological result of lymphadenitis in CSD was of relatively uniform density on CT or homogeneous signal intensity on both T1WI and T2WI, with homogeneous, moderate enhancement following intravenous contrast administration. The corresponding histological analysis revealed that there were numerous granulomas, with proliferation of histiocytes, lymphoid follicles and neutrophilic aggregation. We hypothesized that homogeneous, moderate enhancement corresponded with inflammatory granulomas, and that they were indicative of the early stage of infection without marked necrosis, which was consistent with previous reports $(20,30)$, as they were observed histologically in $8(47.1 \%)$ nodes in the present study. As for the intermediate stage, although it was difficult to differentiate CSD at this point from other chronic granulomatous diseases using radiological data (29), if associated with the special disease history of cat exposure, CSD may also be diagnosed correctly at an early time-point.

The less common radiological result of lymphadenitis in CSD from the present study was exhibited as heterogeneous low-density on the CT scans, heterogeneous isointensity to muscle with astral hypointense areas on T1WI and heterogeneous hyperintensity with an astral hypointense area on fat-suppressed T2WI, which was demonstrated in 4 (23.5\%) nodes. Following intravenous contrast administration, there were 2 different enhancement patterns in these nodes: i) Heterogeneous enhancement, with marked enhancement of the astral hypointense areas; and ii) heterogeneous enhancement with a 'rose flower' sign enhancement of the astral hypointense area. The corresponding histological analysis demonstrated that the inflammatory and necrotic regions were replaced or surrounded by spindle collagen-producing cells, which were composed of variable amounts of fibroblasts or fibrocytes, and we hypothesized that these 2 different enhancement patterns corresponded to the astral low-density/hypointensity area of the nodes composed of variable amounts of fibroblasts or fibrocytes. In cases where immature fibroblasts were the primary component of the astral low-density/hypointensity area, marked enhancement was observed, and in cases where the mature fibrocytes were the primary component in the center surrounded by the fibroblasts, there was no enhancement in the center surrounded by peripheral flower ring reinforcement, indicating that it belonged to a histologically-determined late disease stage. This stage exhibited marked characteristics in the radiological data, particularly the 'rose flower' sign, which had not been described previously in the English language literature, to the best of our knowledge.

Imaging data in CSD usually also demonstrates extensive surrounding edema in the area of lymphatic drainage proximal to the site of inoculation (15). In particular, MRI T2WI images exhibited surrounding edema more clearly compared with CT scans, which was a characteristic of inflammatory lesions, generally not appearing in benign tumors and lymphoma.

For differential diagnosis, the most important factor of cat exposure and imaging features of astral low-density/hypointensity area in enlarged lymph nodes with clear enhancement or the 'rose flower' sign associated with the general subcutaneous edema in the vicinity of the nodes may differentiate CSD from soft-tissue hemangioma, neurogenic tumors lymphoma, metastasis and soft-tissue sarcoma, although it is difficult to form the diagnosis of CSD without a history of cat exposure.

The lymphadenitis in CSD often resolves spontaneously within 3 months. Antibiotics are not indicated in the majority of cases, but they may be considered for severe or systemic disease involving the bone marrow, liver, spleen, brain and eyes (31-35), particularly in immunocompromised patients. A total of $10-35 \%$ of the infected nodes progress to suppuration, and evacuating the pus is necessary in this condition (36).

In summary, the diagnosis of CSD should be considered in relatively young patients with lymphadenopathy in the upper extremities or head and neck region who also exhibit i) astral low-density/hypointensity areas with marked enhancement or the 'rose flower' sign on CT/MRI scans in the late stages of infection, or ii) homogeneous, moderate enhancement on MRI scans in the early stages of infection, or iii) heterogeneous enhancement with non-enhancing areas on $\mathrm{CT} / \mathrm{MRI}$ scans or heterogeneous enhancement with progressively 'spoke-wheel-like' enhancement of the central patchy low-density area on CT scans in the intermediate stages of infection, in single or multiple enlarged lymph nodes associated with general subcutaneous edema in the vicinity of the nodes on CT/MRI. This should be in addition to a history or a renewed anamnesis of exposure to a cat, therefore avoiding unnecessary surgical resection of this pseudotumor. MRI scans were more advantageous compared with $\mathrm{CT}$ in displaying the imaging features in CSD, particularly the characteristics of 
marked enhancement of an astral hypointensity area or the 'rose flower' sign.

\section{References}

1. Regnery RL, Olson JG, Perkins BA and Bibb W: Serological response to 'Rochalimaea henselae' antigen in suspected cat-scratch disease. Lancet 339: 1443-1445, 1992.

2. Rostad CA, McElroy AK, Hilinski JA, Thompson MP, Drew CP Denison AM, Zaki SR, Mahle WT, Rogers J, Abramowsky CR and Shehata B: Bartonella henselae-mediated disease in solid organ transplant recipients: Two pediatric cases and a literature review. Transpl Infect Dis 14: E71-E81, 2012.

3. Rozmanic V, Banac S, Miletic D, Manestar K, Kamber S and Paparic S: Role of magnetic resonance imaging and scintigraphy in the diagnosis and follow-up of osteomyelitis in cat-scratch disease. J Paediatr Child Health 43: 568-570, 2007.

4. Klotz SA, Ivans V and Elliott SP: Cat-scratch disease. Am Fam Physician 83: 152-155, 2011.

5. Nelson CA, Moore AR, Perea AE and Mead PS: Cat scratch disease: U.S. Clinicians' experience and knowledge. Zoonoses Public Health, Jul 14, 2017 (Epub ahead of print).

6. Sander A, Posselt M, Oberle K and Bredt W: Seroprevalence of antibodies to Bartonella Henselae in patients with cat scratch disease and in healty controls: Evaluation and comparison of two commercial serological tests. Clin Diagn Lab Immunol 5: 486-490, 1998

7. De Keukeleire S, Geldof J, De Clerck F, Vandecasteele S, Reynders $\mathrm{M}$ and Orlent M. Prolonged course of hepatic granulomatous disease due to Bartonella henselae infection. Acta Gastroenterol Belg 79: 497-499, 2016.

8. Wear DJ, Margileth AM, Hadfield TL, Fishcer GW, Schlagel CJ and King FM: Cat scratch disease: A bacterial infection. Science 221: 1403-1405, 1983.

9. Margileth A: Recent advances in diagnosis and treatment of cat scratch disease. Curr Infect Dis Rep 2: 141-146, 2000.

10. Rolain JM, Brouqui P, Koehler JE, Maguina C, Dolan MJ and Raoult D: Recommendations for treatment of human infections caused by Bartonella species. Antimicrob Agents Chemother 48: 1921-1933, 2004.

11. Schutze GE: Diagnosis and treatment of Bartonella henselae infections. Pediatric Infect Dis J 19: 1185-1187, 2000.

12. Bass JW, Vincent JM and Person DA: The expanding spectrum of Bartonella infections: II. Cat-scratch disease. Pediatr Infect Dis J 16: 163-179, 1997.

13. Florin TA, Zaoutis TE and Zaoutis LB. Beyond cat scratch disease: Widening spectrum of Bartonella henselae infection. Pediatrics 121: e1413-e1425, 2008.

14. Margileth AM: Recent advances in diagnosis and treatment of cat scratch disease. Curr Infect Dis Rep 2: 141-146, 2000

15. Wang CW, Chang WC, Chao TK, Liu CC and Huang GS Computed tomography and magnetic resonance imaging of cat-scratch disease: A report of two cases. Clin Imaging 33: 318-321, 2009

16. Jackson LA, Perkins BA and Wenger JD: Cat scratch disease in the United States: An analysis of three national databases. Am J Public Health 83: 1707-1711, 1993.

17. Nelson CA, Saha S and Mead PS. Cat-scratch disease in the United States, 2005-2013. Emerg Infect Dis 22: 1741-1746, 2016

18. Dong PR, Seeger LL, Yao L, Panosian CB, Johnson BL Jr and Eckardt JJ: Uncomplicated cat-scratch disease: Findings at CT, MR imaging, and radiography. Radiology 195: 837-839, 1995.
19. Erbe $\mathrm{M}$ and Lindenfelser R: Solitary nontuberculous mycobacterial infection of a cervical lymph node caused by Bartonella henselae (cat scratch disease). Mund Kiefer Gesichtschir 8: 381-386, 2004 (In German).

20. Gielen J, Wang XL, Vanhoenacker F, De Schepper H, De Beuckeleer L, Vandevenne J and De Schepper A: Lymphadenopathy at the medial epitrochlear region in cat-scratch disease. Eur Radiol 13: 1363-1369, 2003.

21. Parinaud $\mathrm{H}$ : Conjonctivite infectieuse paraissant transmise a l'homme par les animaux. Soc Ophthalmol Paris 2: 29-31, 1889.

22. Psarros G, Riddell J IV, Gandhi T, Kauffman CA and Cinti SK: Bartonella henselae infections in solid organ transplant recipients: Report of 5 cases and review of the literature. Medicine (Baltimore) 91: 111-121, 2012

23. Zangwill KM, Hamilton DH, Perkins BA, Regnery RL, Plikaytis BD, Hadler JL, Cartter ML and Wenger JD: Cat scratch disease in Connecticut. Epidemiology, risk factors, and evaluation of a new diagnostic test. N Engl J Med 329: 8-13, 1993.

24. Carithers HA: Cat-scratch disease. An overview based on a study of 1,200 patients. Am J Dis Child 130: 1124-1133, 1985.

25. Margileth AM: Dermatologic manifestations and update of cat scratch disease. Pediatr Dermatol 5: 1-9, 1988.

26. Bass JW, Vincent JM and Person DA: The expanding spectrum of Bartonella infections: II. Cat-scratch disease. Pediatr Infect Dis J 16: 163-179, 1997.

27. Verçoza AM, de los Santos CA and Vargas JA: Cat scratch disease in kidney transplant receptors: Is it a rare or underdiagnosed pathology? J Bras Nefrol 36: 406-409, 2014.

28. Margileth AM: Cat-scratch disease. Adv Pediatr Infect Dis 8: $1-21,1993$.

29. Nwawka OK, Nadgir R, Fujita A and Sakai O: Granulomatous disease in the head and neck: Developing a differential diagnosis. Radiographics 34: 1240-1256, 2014

30. Mele FM, Friedman M and Reznik AM: MR imaging of the knee: Findings in cat-scratch disease. Am J Roentgenol 166 1232-1233, 1996.

31. Al-Rahawan MM, Gray BM, Mitchell CS and Smith SD: Thoracic vertebral osteomyelitis with paraspinous mass and intraspinal extension: An atypical presentation of cat-scratch disease. Pediatr Radiol 42: 116-119, 2012

32. Thudi KR, Kreikemeier JT, Phillips NJ, Salvalaggio PR, Kennedy DJ and Hayashi PH: Cat scratch disease causing hepatic masses after liver transplant. Liver Int 27: 145-148, 2007.

33. Mortelé KJ, Segatto E and Ros PR: The infected liver: Radiologic-pathologic correlation. Radiographics 24: 937-955, 2004.

34. Roebuck DJ: Cat-scratch disease with an extraaxial mass. AJNR Am J Neuroradiol 19: 1294-1295, 1998.

35. Tolou C, Mahieu L, Martin-Blondel G, Ollé P, Matonti F, Hamid S, Benouaich X, Debard A, Cassagne M and Soler V: Posterior segment involvement in cat-scratch disease: A case series. J Fr Ophtalmol 38: 974-982, 2015.

36. Meier JD and Grimmer JF: Evaluation and management of neck masses in children. Am Fam Physician 89: 353-358, 2014.

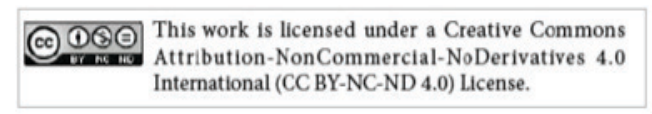

